\title{
Practice guidelines and standards of care in mental health care
}

\author{
Jan Spijker ${ }^{1}$, Janneke Wentink-van de Laar ${ }^{2}$, Anneke Huson ${ }^{3}$, and Aart Schene ${ }^{4}$ \\ ${ }^{1}$ Pro Persona Mental Health Care, \\ ${ }^{2}$ Alliance Quality in mental health care (Akwa GGZ), \\ ${ }^{3}$ Dutch Depression Association \\ ${ }^{4}$ Radboudume
}

May 5, 2020

\begin{abstract}
Aims Clinical guidelines have made an impact on the quality of care but recently their drawbacks became more visible. In this article standards of care are introduced as a new and further step in de development of clinical guidelines in the Netherlands Methods An historical overview of guideline and care standard development and an example of the standard for depressive disorders are presented Results Standards of care describe in general terms from the perspective of the patient what good care is for people with a certain mental disorder during the entire care continuum, the patient journey. They describe for all parties in the mental health care system what is necessary in the care process. Generic standards describe healthcare components or healthcare topics that are relevant to multiple mental disorders More than 40 standards of care/generic standards have been developed. Conclusions Standards of care seem to be a solution for the problem of different and sometimes conflicting monodisciplinary guidelines, the limited scope of the monodisciplinary guidelines and the omission of clinical expertise and patients preferences in the clinical guidelines. However implementing the new set of comprehensive standards of care will be a challenge.
\end{abstract}

\section{Introduction}

Improving quality of care by using clinical guidelines based on well graded research evidence has been the standard in the Netherlands for the past 25 years, also in mental health care. In these years we have gradually improved our knowledge about how to evaluate health and health care related scientific results. By trial and error we learned how to meta-analyse and integrate ever increasing amounts of scientific findings into applicable scientific knowledge. We also discovered how to develop, publish and disseminate clinical guidelines and how to educate professionals in using guidelines as tools for the implementation of new clinical practices. Comparing the training of mental health professionals today with that of the pre millennial nineties shows a tremendous paradigm shift from opinion expert based clinical thinking and practicing towards structured, protocoled, multidimensional, evidence based practice.

However, after this first promising early age a possibly too strict and consistent utilization of evidence based medicine more recently begins to show some of its drawbacks and possible disadvantages more clearly. Questions about the further progress from 'one disorder' guidelines towards guidelines for complex and comorbid health conditions ${ }^{1,2}$, the appraisal and integrated use of more diverse types of knowledge (observational, expert, experiential $)^{3}$, about the financial consequences of implementing guidelines for health care costs, about the real effectiveness of guideline implementation for changing provider performance and/or patient outcomes $^{4}$, and about the principles for updating of guidelines ${ }^{5}$ threaten a productive further progress.

Different countries have shown different evidence based practices and guideline histories and are trying to find cultural sensitive solutions for the above named questions. In this paper we will describe the development and current situation in Dutch mental health care. We aim to consider three aspects: We first give information 
about the different guideline programs that were developed over the past two decades; what steps have been taken, how did we organize this, what was the output, and what were the limitations. Second, we will elaborate on this development in more detail and focus on the past five years during which we executed a nationwide program to produce a set of practice guidelines (standards of care and generic standards). The program was commissioned by the Dutch Foundation for Quality Development (NKO). We describe how and by whom NKO was created and how this program resulted in a set of quality standards. Third, we want to describe an example (the standard of care for depression) to give more detail about the way we have been working. We value sharing our experiences with practice guidelines program for mental health care and hope it will be inspiring for other countries.

\section{Dutch mental health care}

In the Netherlands mental health care services are provided by public organizations which are divided over three compartments: primary care, generalized mental health care and specialized mental health care. Over the past ten years the nationwide policy was focused on a further transition from specialized care to generalized care and even primary care, including a rather ambitious reduction of clinical inpatient services $(30 \%)$ and a further development of community-based outpatient care, which had to integrate health care and social care in patients own environment as much as possible.

At this moment mental health spending in the Netherlands is $8 \%$ of overall health care spending, which is the median of a recent survey in 14 different western countries ${ }^{6}$

\section{Development of guideline programs}

Parallel to the introduction of clinical guidelines in mental health care in the US and the UK, the first mental health guideline in the Netherlands was introduced on the topic of Depression in 1994. Many other guidelines were initiated by various professional associations in the following years. In 1998 these professional associations joined together on a national level by starting a ten years multidisciplinary guideline development program, financed by the Ministry of Health. Aim of this program was to produce a set of multidisciplinary mental health guidelines and to prevent further diversification of monodisciplinary documents. In ten years time this collaboration delivered a total of 12 multidisciplinary guidelines for most psychiatric disorders. This period of multidisciplinary guideline development came to an end in 2009 due to the loss of funding. In addition to these multidisciplinary guidelines, the Dutch Psychiatric Association (DPA) also produced 15 monodisciplinary mental health guidelines. In some of these guidelines the DPA worked together with other medical specialties (eg neurology, internal medicine, anaesthesiology).

Increasing health care costs combined with failing economic development changed the political focus during the first decade towards (mostly somatic) chronic diseases. Related to that development new concepts like care programming, disease management and integrated care were introduced. Within these new approaches standards of care were introduced as multidisciplinary documents which described what good care for a specific chronic disease or disorder should minimally include, seen from the patients point of view. These standards of care had to find their societal impact in close collaboration with the clinical guidelines.

In 2010, standards of care were introduced for eg diabetes, heart failure, arthrosis and COPD, and the mental health field produced one such product, a concept standard of care for depression. The value of the different types of knowledge that were used in the standard of care for depression (scientific, expert opinion, patient perspective) was unclear. This was one of the reasons the document was not accepted by the professional organizations.

In 2013 a new nationwide initiative was born, the Dutch Foundation for Quality Development (NKO, now Akwa GGZ*11* The Dutch Foundation for Quality Development (NKO) is now Alliance Quality in mental health care (Akwa GGZ) since January 2019.). The NKO received a budget of 15 million euro's from the Ministry of Health to develop a large number of practice guidelines for mental disorders in a five year period. The NKO is an initiative by the Dutch society of Psychologists, the DPA and the Dutch Federation of Patient and Family Associations in Mental Healthcare. More than 1000 professionals (representing 80 
different associations of professionals), patients, care providers and financers joined forces and have been working together in the development of these standards.

\section{Explanation of quality standards}

Clinical guidelines, standards of care and generic standards are all 'practice guidelines'. According to the definition of the Healthcare Institute in the Netherlands, a practice guideline describes in general terms what we define as good healthcare from a patients point of view. These practice guidelines are based on the most actual knowledge, which includes scientific knowledge as well as practice based knowledge and patient preferences.

A standard of care describes in general terms from the perspective of the patient what good care is for people with a certain mental disorder during the entire care continuum or the patient journey (early detection and prevention, diagnostic assessment and monitoring, treatment and recovery, re-integration an participation). A standard of care sets the (national) standard that multidisciplinary, integrated care for mental disorders must meet. In addition, the standard of care states what the patient can expect, not only concerning medication and psychosocial treatment, but also when it comes to societal participation, help in the community and the organization of care. A standard of care describes for all parties in the mental health care system what is necessary in the care process.

Where a standard of care relates to one specific mental disorder, a generic standard describes healthcare components or healthcare topics that are relevant to multiple mental disorders. For example, topics such as self-management, support for relatives, and participation in labour or other activities. A generic standard describes care in different phases of the care process.

A single standard of care may be based on multiple clinical guidelines and can refer to other relevant clinical guidelines. So, standards of care and generic standards are based on existing clinical guidelines but also on new scientific evidence, practice based knowledge and patients preferences. These standards and the underlying guidelines thus form an integral document that enables the care provider and the patient to become acquainted with the intended content and the organization of the care process; and subsequently to assess its practical application.

The following principles have been applied in the development of practice guidelines for mental health care in the Netherlands:

- Perspective of the patient (and his relatives) is central.

- Matched care.

- Shared decision making.

- The right care, in the right place, by the right person, at the right time.

- No more care than necessary and no less care than necessary.

\section{Current practice guideline program in the Netherlands}

NKO has a board (in which the 3 founding parties were represented), an agenda council (for advising the board on substantive programming) and an implementation council (for advising the board on implementation issues).

NKO financed the development of all practice guidelines: each standard had a contractor, often a third party for project management. Practice guidelines are developed involving stakeholders who contribute through participation in a working group, consultation and authorization. Stakeholders include patients and their relatives, professionals, but also representatives of healthcare providers and financers. Each standard had a development phase (1.5 years on average), a consultation phase (3 months on average) and an authorization phase (also 3 months on average).

Between 2014 and 2018 NKO developed over 40 practice guidelines (standards of care (table 1) and generic standards (table 2) for Dutch mental health care. Development and structure of the practice guidelines was identical and all standards were edited and legally checked. In June 2019 a large number of practice 
guidelines were included in the Register of the Healthcare Institute of the Netherlands. This is an important milestone because in the Netherlands this Register is the central place for information about quality of care*11* The registration of a quality standard or measuring instrument in the Register does not change the responsibilities of a healthcare provider vis-à-vis a client. Whether or not a quality standard or measuring instrument has been drawn up with perseverance from the Healthcare Institute, or whether it is included in the Register, has no legal consequences for the legal relationship between the care provider and the client. The Healthcare Inspection can use the registered quality standards as a basis for monitoring and maintaining the quality of care provision. The Healthcare Inspection thus has a growing set of field standards for quality of care. The Healthcare Inspection involves both field standards from the Register and field standards that are not (yet) registered in the Register in its supervision..

\section{Standard of care Depressive symptoms and disorders}

The standard of care depressive disorders addresses depressive disorders (including the persistent depression) according to the DSM-5 as well as depressive symptoms (minor depression or subthreshold depression). The standard of care refers to youth (from the age of 8 years), adults and elderly.

\section{Procedure}

The process of developing the standard of care depressive disorders started with the formation of the working group with delegates of all relevant stakeholders: professionals working in primary, general and specialized health care and the patients and family representatives. An advisory board was installed with the relevant stakeholders including representatives of insurance companies. The development process was supported by researchers of the Netherlands Research Institute for Mental Health.

As a first step, the existing clinical guidelines and comparable documents to improve care for depressed patients were studied. The most important one is the Dutch multidisciplinary guideline for depression ${ }^{7}$ and its addenda for the elderly and the young.

Important elements that were missing or were outdated in this multidisciplinary guideline were notified and selected. The topics were prioritized and a selection was made as not all topics could be addressed for time reasons. This was done by professionals and patients. Persistent depression (including dysthymia) and the effects of food and nutritional supplements in the treatment of depression were prioritized. An update of the guideline was performed for these topics according to the evidence-based guideline development procedure, ${ }^{8}$.

At the same time a survey was carried out among members of patients association for depression in the Netherlands to assess the needs of patients with regard to relapse prevention of depression. A focus group meeting was organized for patients to address the key issues from the patients perspective in the care for persistent depression.

The development of the standard consisted of several meetings with the working group to discuss the existing clinical guidelines for depression, that were summarized by the researchers. An preliminary text of the standard was presented as a working document. New information from the updates and the survey was discussed in the working group. After consensus was reached, it was integrated in the standard. A concept version of the standard was commented upon by the advisory board and subsequently send to all the participating organizations for comments and finally authorization.

\section{Important topics of the standard of care}

There are several topics in the standard of care that are considered as important elements of good clinical care for patients with depression and were not (or differently) addressed in the multidisciplinary guideline depression. These topics are a combination of scientific evidence, practice based knowledge and patients preferences.

Recovery, participation and re-integration should be addressed from the start of treatment. Personal wellbeing, coping with depression and quality of life are essential elements for patients. Empowerment, selfmanagement skills and self-direction are steps that help to overcome depression. To reach personal recovery, 
re-integration in (volunteer) work and/or social activities is crucial. Some limitations in functioning may persist after treatment of depression and finding the balance between capacity and stress is an important challenge. Support in daily and weekly structuring might be necessary and methods from rehabilitation practices can be helpful for this purpose. Peer support can be helpful to reach recovery and options to contact experiential experts should be promoted. Mental health professionals and experiential experts should work together to promote peer support facilities in each region.

Support for the social network. Supporting family and relatives of patient can enhance and consolidate the effects of treatment. Psycho-education, emotional support, teaching coping styles and practical tips are elements of support for the network.

Matched care is the preferred option to organize treatment for depression. Treatment options for depression are numerous and matching treatment to patients preferences and characteristics is crucial for treatment success. Matched care means generalist and accessible care if possible and specialized and intensive care if needed. Matching to specific interventions or a specific professional is preferred, taking nature, severity and course of depression into account.

Restricted use of antidepressants is warranted. There is a growing awareness of the high usage of antidepressants in the population. Professionals and patients recognize the importance to reduce the prescribing of antidepressants. Before antidepressants are prescribed professional and client should consider nonpharmaceutical options with strict agreements on monitoring and evaluation of the treatment plan. It is important to take note that the efficacy of antidepressants is limited compared to placebo in non-severe depression and effects of antidepressants do not endure after cessation of the treatment. Side effects of antidepressants are problematic and discontinuation of treatment can result in withdrawal symptoms.

Treatment for persistent depression . Preferred treatment for persistent depression is a combination of biological treatment and evidence based psychotherapy. Knowledge from practice and patients preferences indicate that more sessions of psychotherapy might be needed than is stipulated in the protocols and also that psycho-analytic psychotherapy is an optional intervention, even though the scientific evidence is weak.

Relapse prevention . Patients with depression express an urgent need to address relapse prevention. Important themes for patients are the accessibility of care in case of an emergent relapse and the option to contact a professional even when treatment has ended. A relapse prevention plan is mandatory for every patient who terminates treatment.

Food and nutritional supplements. Especially patients representatives supported the update of the evidence on the effects of food and nutritional supplements in the treatment of depression. The evidence for vitamin supplements, zinc-supplements, medicinal herbs (excluding St Johns worth), reed ginseng and tryptophan is weak and/or contradictory. Only omega-3 polyunsurated fatty acids are found to be effective in the treatment of depression in adults.

Arts therapies Professionals and patients representatives highly appreciate arts therapy (music therapy, creative therapy, dance therapy etc) for depression. Therefore it is stated that arts therapy can be part of every treatment plan as an adjunct to the evidence based treatment interventions. The indication for these interventions is the result of a shared decision making process.

\section{Discussion}

In this article we described the development from evidence based clinical guidelines to standards of care and generic standards for mental health care in the Netherlands over the last decades and presented the standard of care for depressive disorders as an example.

In the present situation there are more than 40 standards of care/generic standards that have been authorized by all relevant professional organizations. The standards of care addresses specific mental disorders while the generic standards refer to overlapping themes in clinical practice. The generic standards connect the different 
standards of care. This complex matrix structure of standards encompasses the whole field of mental health care and describes good clinical care from the perspective of patients with mental disorders in all aspects.

This process of the development of these standards was ambitious and as far as we know worldwide unique. Several important discussions were raised on the way: How to integrate scientific evidence with clinical expertise and experiential knowledge? And how to implement more than 40 standards?

\section{How to integrate different sources of knowledge}

Despite the success of guidelines in providing more rational and effective health care, the use of guidelines has recently been criticized as being impersonal and leaving little room for expertise of the clinician and the patients views and preferences ${ }^{9}$. Clinical expertise might enrich scientific evidence as clinicians know what actually works in practice. The experiential knowledge of patients could improve the quality of care because patients bring in the unique experience how to life with a disorder ${ }^{10}$. The combination of scientific evidence with clinical expertise and patients preferences also might stimulate the acceptability and implementation of guidelines ${ }^{11}$.

However, no coherent method has been developed thus far to integrate scientific evidence with clinical expertise and experiential knowledge. For scientific evidence the GRADE system ${ }^{12}$ has been developed, a common, sensible and transparent approach to grade quality (or certainty) of evidence and strength of recommendations. For research on clinical expertise and experiential knowledge different research methods are used (participatory research, qualitative research, narratives), but their scientific base is not as strong as in evidence based medicine. Furthermore, a procedure how to weigh each domain of knowledge is currently lacking.

The developmental process of the standard of care for depressive disorders showed how clinical expertise and experiential knowledge supplemented scientific evidence in cases where evidence was weak or lacking. Interesting here is the adoption of art therapies in this standard of care although the evidence for the efficacy of art therapies is currently lacking. Psycho-analytic psychotherapy is added as an optional psychotherapeutic method, despite a weak scientific base, because of the strong appreciation of professionals for this form of psychotherapy, at least in our country. Methods to assemble experiential knowledge were a survey among members of the consumers organization and a focus group meeting. Consensus meetings in the working committee were used to weight the recommendations that came from the different perspectives and that method proved effective but is of course not fully transparent. It is important to develop an general accepted procedure to integrate scientific evidence, clinical expertise and experiential knowledge in practice guidelines.

\section{Implementation of care standards}

When a multitude of guidelines were introduced in the field of mental health in the past decades, implementation proved to be a slow and difficult process. A multi-faceted approach is mostly promoted as the best strategy with actions targeted at the professional level (training programs, educational material, access to peer information about good practice experiences), the organizational level (introduction of care pathways, tools for cost estimation) and the patient level (information brochures or option grids). A multitude of resources and tools to help to implement guidelines has been developed but implementation is still work in progress. Continuous efforts and financial incentives are necessary to optimize the use of guidelines ${ }^{13}$. Whether improved adherence to guidelines, will improve quality of care and patients outcome is open to debate and a little researched question ${ }^{4}$.

Although the implementation of the number of standards seems to be an obstacle for some, it can also be an advantage. There might be some advantages with implementation of the standards compared to implementation of guidelines. The introduction of elements of clinical expertise in the standards might enthuse clinicians more than before to adapt to these standards as they represent the daily clinical practice better. At the same time, patients will recognize more elements of experiential knowledge in the standards and the associations of patients representatives might enforce the use of these standards much stronger than the guidelines before. 
Where NKO previously supported the field in the development of standards, Akwa GGZ ${ }^{14}$ now supports mental health care on a national level with the implementation of these standards. For all standards of care a test was carried out among clinical practitioners to assess the practicability of the care standard and the conditions that are necessary to implement the care standard. With that information Akwa GGZ has developed implementation plans that includes strategies to inform professional organizations how to use the standards; there is an online database where all standards can be found and searched; all standards have been translated to version for users which are on-line, there is an toolkit with strategies to help the implementation and Akwa GGZ will monitor the process of implementation of the standards. This nationwide, encompassing and structured program for implementation might help the implementation process further. But the professional organizations are responsible for the actual implementation. Insurance parties probably will be an active promotor of implementation as the care standards do represent the best care as possible. The goal is that professionals, patients and their families know and use the practice guidelines in daily practice; that professionals and providers learn from each other by exchanging information and that the quality of care in Dutch mental health care as a whole will improve.

In conclusion, the process of the development of care standards and generic standards in the Netherlands is an important next phase in the ongoing challenge to improve the quality of care for mental health disorders with evidence based medicine. Some of the difficulties that arose in the guideline development in the early phase like the multitude of different and sometimes conflicting monodisciplinary guidelines, the limited scope of the monodisciplinary guidelines and the omission of clinical expertise and patients preferences in the guidelines are addressed by this nationwide, multidisciplinary, integrated new set of standards of care and generic standards, all developed by the same methodology with a strong influence of patients and families, and acceptance by the different health care insurance companies. Still, there are important challenges for the future because the consequences of implementation of the standards (for example the financial consequences) are not fully identified. Furthermore, the question how to update the standards of care is as relevant as it was for the clinical guidelines ${ }^{5}$. Finally, the efforts and expenses for the development and implementation of these standards can only be justified, if it really improves the care for those with mental disorders.

\section{Table 1: Standards of care}

1. Standard of care Adjustment disorder (including burn-out)

2. Standard of care Attention deficit hyperactivity disorder

3. Standard of care Anxiety symptoms and disorders

4. Standard of care Autism

5. Standard of care Bipolar disorders

6. Standard of care Conversion disorder

7. Standard of care Depressive symptoms and disorders

8. Standard of care Dissociative disorders *

9. Standard of care Eating disorders

10. Standard of care Opiate addiction

11. Standard of care Paraphilic and hypersexual disorders

12. Standard of care Personality disorders

13. Standard of care Problematic alcohol use and alcohol addiction

14. Standard of care Psychosis

15. Standard of care Psychotrauma*

16. Standard of care Sexual dysfunctions

17. Standard of care Somatic symptom disorders

18. Multidisciplinary guideline: Disorders in the use of cannabis, cocaine, amphetamine, ecstasy, GHB and benzodiazepines

* This standard of care is in development

Table 2 Generic standards 
1. Generic standard Acute psychiatry

2. Generic standard Work as a medicine

3. Generic standard Adverse effects

4. Generic standard Comorbidity

5. Generic standard Daily activities and participation

6. Generic standard Destigmatization

7. Generic standard Diagnostics and treatment of suicidal behaviour

8. Generic standard Diversity

9. Generic standard Coercion

10. Generic standard eHealth

11. Generic standard Severe mental disorders

12. Generic standard Diagnostics and treatment in general basic mental health care

13. Generic standard Recovery support

14. Generic standard Collaboration agreements for youth mental health care

15. Generic standard Collaboration agreements between general practitioner, general basic mental health care and specialized mental health care

16. Generic standard Organization of care for child and youth

17. Generic standard Elderly people with mental illness

18. Generic standard Psychological complaints in general practice

19. Generic standard Psychiatric complaints in childhood

20. Generic standard Psychiatric disorders and (mild) intellectual disability

21. Generic standard Psychotherapy

22. Generic standard Collaboration and support for relatives of people with psychological problems

23. Generic standard Auditory hallucinations

24. Generic standard Arts therapy

25. Generic standard Early detection of psychological complaints

26. Generic standard Self-management

27. Generic standard Hospital psychiatry

\section{Literature}

1. Harding E, Pettinari CJ, Brown D, Hayward M, Taylor C. Service user involvement in clinical guideline development and implementation: learning for mental health service users in the UK. Intern Rev Psychiatr 2011; 23 (4): 352-357

2. Salvador-Carulla L, Lukersmith S, Sullivan W From the EBM pyramid to the Greek temple: a new conceptual approach to Guidelines as implementation tools in mental health. Epidem Psychiatr Scienc 2017; 26(2): 105-114.

3. Wieringa S, Dreesens D, Forland F, Hulshof C, Lukersmith S, Macbeth F, Shaw B, van Vliet A, Zuiderent-Jerak T; AID Knowledge Working Group of the Guidelines International Network. Different knowledge, different styles of reasoning: a challenge for guideline development. BMJ Evid Bas Med 2018; 23(3): 87-91.

4. Girlanda F, Fiedler I, Becker T, Barbui C, Koesters M. The evidence-practice gap in specialist mental healthcare: a systematic review and meta-analysis of guideline implementation studies. Br J Psychiatr 2017; 210: 24-30.

5. .Shekelle PG. Clinical Practice Guidelines. What's next? JAMA 2018; 28: 757-758.

6. NHS Benchmarking Network) . International Mental health Comparisons. Adult and older adult services. 2018

7. Spijker J, Bockting CLH, Meeuwissen JAC, Vliet IM van, Emmelkamp PMG, Hermens MLM, Balkom van ALJM namens de Werkgroep Multidisciplinaire richtlijnontwikkeling Angststoornissen/Depressie. Multidisciplinaire richtlijn Depressie (Derde revisie). Richtlijn voor de diagnostiek, behandeling en begeleiding van volwassen patiënten met een depressieve stoornis. 2013 Utrecht: Trimbos-instituut.

8. AGREE Collaboration. Development and validation of an international appraisal instrument for asses- 
sing the quality of clinical practice guidelines: the AGREE project. Qual Safe Health Care 2003; 12: $18-23$.

9. Weaver RR . Reconciling evidence based medicine and patient centered care: defining evidence based inputs to patients-centered decisions. J Evaluat Clin Pract 2015; 21 (6): 1076-1080.

10. Pols J. Knowing patients. Scienc Tech Hum Val 2014; 39 (1): 73-97

11. Harder T, Abu Sin M, Bosch-Capblanch X, Bruno Coignard, de Carvalho Gomes H, Duclos P et al. Towards a framework for evaluating and grading evidence in public health. Health Pol 2015; 119(6): $732-6$

12. GRADE website. http://www.gradeworkinggroup.org.

13. Moreno EM, Moriana JA Clinical guideline implementation strategies for common mental health disorders. Rev Psiq Sal Ment 2016; 9(1): 51-62.

14. Akwa GGZ Zorgstandaarden GGZ. www.ggzstandaarden.nl 